\title{
Karoline Pichlers Denkwürdigkeiten: Ein Selbstbekenntnis?
}

\section{Antonie Alm-Lequeux}

Die Wiener Schriftstellerin Karoline Pichler (1767-1843) ist heute nahezu vergessen. In österreichischen Literaturgeschichten taucht ihr Name zwar noch vereinzelt auf, ihr umfangreiches Werk wird jedoch zugunsten ihres "literarischen Salons", in dem in den ersten Jahrzehnten des neunzehnten Jahrhunderts Gelehrte und Dichter von Rang und Namen verkehrten, nur selten erwähnt.

Schon zu Pichlers Lebzeiten fanden die Abendgesellschaften mehr Anerkennung als ihre schriftstellerische Tätigkeit. So bezeichnete der Schwede Per Daniel Atterbom den Pichlerschen Salon (neben dem Stephansdom) als eine der "Hauptmerkwürdigkeiten" Wiens. Und obwohl ihre Schriften zu einer Zeit "als der schönste Schmuck (!) in jeder Privatbibliothek" galten, ${ }^{1}$ blieben ästhetische Urteile über ihr Werk weitgehend aus. Goethe war einer der wenigen Zeitgenossen, die sich konstruktiv über einen Roman geäußert haben. An eine gemeinsame Freundin schrieb er, den Briefroman Agathokles nicht nur "mit Aufmerksamkeit und vielem Vergnügen" gelesen zu haben, er lobte auch "die Zeichnung der Charaktere, die Anlage und die Durchführung" ${ }^{2}$ des Romans. Wesentlich häufiger findet man jedoch Aussagen wie die folgende von einem Hofrat Reinbeck, der in seinen Reiseaufzeichnungen bemerkte:

${ }^{1}$ Adolf Bäuerle (Hg.): Allgemeine Theaterzeitung und Originalblatt für Kunst, Literatur, Musik, Mode und gesellschaftliches Leben, Wien 1843. In: Emil Blümml (Hg.): Denkwürdigkeiten aus meinem Leben, 2 Bde. München 1914, S. XXIX.

${ }^{2}$ Goethe am 30. August 1812 an Eleonore Flies. Weiter bemerkte Goethe: "'[...] Die innere Konsequenz des Werkes hat mich mit allem, was mir sonst hätte fremd bleiben müssen, wirklich befreundet.' Obwohl er den Plan hier und da umdenken, 'einen Charakter eine andere Richung, einer Begebenheit eine andere Wendung' erteilen wollte, so wurde er immer durch die Verfasserin in der Folge bekehrt und 'auf ihren eigenen Sinne zurückgebracht', so $\mathrm{da} ß$ er die Arbeit in jedem Sinne gegen jede Einwendung in Schutz nehmen könnte [...]." Blümml: Denkwürdigkeiten I, S. 629. 
Eine edlere Weiblichkeit habe ich nie kennen gelernt in der großen Welt. Gilt es den Beweis, wie wenig Berühmtheit in sonst nicht gewöhnlichen weiblichen Sphären den echten weiblichen Charakter zu beeinträchtigen vermag, so findet man ihn in dieser Frau. Wäre ich mit ihrem schriftstellerischen Verdienste nicht bekannt gewesen, ich würde in ihr die liebevolle Gattin und Mutter und die verständige Hausfrau und liebenswürdige Gesellschafterin bewundert haben, ohne vielleicht eine Ahnung davon zu erhalten, daß sie auch ein anderes Streben verfolge, als in diesen häuslichen Sphären sich geltend zu machen. ${ }^{3}$

Reinbeck beschreibt in diesem kurzen Anschnitt Pichlers "edle Weiblichkeit" und geht nicht weiter auf ihr schriftstellerisches Verdienst ein, erstaunlich, wenn man weiter liest, daß von allen literarischen Bekanntschaften jene mit Karoline Pichler ihm die interessanteste geblieben sei. ${ }^{4}$

"Echte und edle Weiblichkeit" bestimmt somit das Lebenswerk einer Frau, deren Dramen mit Erfolg im Burgtheater aufgeführt, deren Erzählungen mehrfach übersetzt wurden, deren historische Romane, Erzählungen, Dramen, Gedichte, Idyllen, Balladen, Aufsätze es auf ein ansehnliches sechzigbändiges Gesamtwerk brachten. Diese auffallende Diskrepanz zwischen Pichlers Schaffen, ihrem Erfolg und dem reservierten und ambivalenten Urteil ihres Publikums legt es nahe, Selbstaussagen heranzuziehen und zu untersuchen, wie dieses Bild entstanden ist, und inwiefern die Wienerin dieses Bild von ihrer "echten Weiblichkeit" möglicherweise selbst konstruiert hat.

Karoline Pichlers letztes und heute bekanntestes Werk sind ihre Denkwürdigkeiten. In dieser 1844 postum veröffentlichten vierbändigen Autobiographie berichtet Pichler neben familiären Ereignissen über ihre literarische Tätigkeit, ihre Gesellschaftsabende, über das politische und kulturelle Leben in Wien. Wiederholt wurden die Denkwürdigkeiten als "historisches Zeitgemälde" bezeichnet, als "kulturgeschichtliche Quelle"

${ }^{3}$ In: Karl Glossy: "Hormayr und Karoline Pichler", Jahrbuch der Grillparzer-Gesellschaft XII (1902), S. $220 \mathrm{f}$.

4 Ibid.

${ }^{5}$ Glossy, S. 212 f. 
angesehen und auszugsweise in Bücher zur österreichischen Kulturgeschichte und auch in Schulbücher aufgenommen.

Emil Blümml, der die Denkwürdigkeiten 1914 mit einer Einleitung und zahlreichen Anmerkungen versehen in der Reihe "Denkwürdigkeiten aus Alt-Österreich" neu herausgab, bezeichnete sie aufgrund ihres Stils und ihres Inhalts als "klassisches Werk der österreichischen Denkwürdigkeitenliteratur". ${ }^{6}$ Damit stellt er Pichler neben Klemens von Metternich, dessen Denkwürdigkeiten in der gleichen Reihe erschienen und viele andere einflußreiche Persönlichkeiten, die in den vierzigerer Jahren ihre Lebensgeschichte unter diesem Titel vor dem Hintergrund der napoleonischen Kriege und der Kongreßzeit veröffentlichten. Die diese Lebensaufzeichnungen bestimmende politische Komponente kommt in Ingrid Aichingers Definition deutlich zum Ausdruck, in der sie "die Bezüge zur Zeitgeschichte und Politik" als Gattungscharakteristikum erklärt. Als solche werden "die vielen Berichte von Kriegserlebnissen, von Schicksalen aus Perioden innenpolitischer Spannungen, Anklage- und Rechtfertigungsschriften von Soldaten, Politikern, Diplomaten, aber auch von politisch engagierten Schriftstellern" angeführt. ${ }^{7}$ Die Definition zeigt, wie sehr die Gattung auf männliche Verfasser zugeschnitten ist.

Aber auch wenn man sich dem Zweig der autobiographischen Schriften zuwendet, der nach der Gattungsdefinition nicht äußerliche Begebenheiten, sondern die innere Entwicklung eines Menschen zum Thema hat, einem Genre also, das der Geschlechtertypologie zufolge der Frau näher liegen sollte - und Pichler gibt vor, ein Selbstbekenntnis geschrieben zu haben finden sich vorrangig männliche Autoren. Der in einer Autobiographie nachgezeichnete und zur Veröffentlichung bestimmte Prozeß der Selbsterkenntnis ist, wie auch das Memoirenschreiben, vor allem Männern vorbehalten gewesen.

In der germanistischen Literaturwissenschaft wurde daher lange davon ausgegangen, daß Frauen keine Autobiographien geschrieben haben, und auch in jüngeren Untersuchungen zur deutschen Autobiographie finden Lebensberichte von Frauen noch keine Beachtung. ${ }^{8}$ Feministische Un-

${ }^{6}$ Blümml, "Einleitung", S. LXXXI.

7

Reallexikon der deutschen Literaturgeschichte, Berlin 1977, Bd. 3, "Selbstbiographie", S. 815.

8

${ }^{8}$ Günter Niggl erwähnt die Beschäftigung mit weiblichen Autobiographien 
tersuchungen haben hingegen herausgestellt, daß Frauen um $1800 \mathrm{zwar}$ Selbstzeugnisse verfaßt, jedoch andere Ausdrucksformen gewählt haben. ${ }^{9}$ Elke Ramm geht so weit von einer "Gattungsverweigerung" zu sprechen, Frauen bevorzugten, da ihnen eine öffentliche Selbstoffenbarung verwehrt war, ihre "Lebensgeschichten in romanesker Form zu thematisieren". ${ }^{10}$ Wohl aus diesem Grund wurden Karoline Pichlers autobiographische Denkwïrdigkeiten weder in der traditionellen noch in der feministischen Autobiographie-Forschung behandelt.

Pichler hat sich in den Denkwürdigkeiten in Titel, Inhalt und Form an die zu ihrer Zeit übliche Schreibpraxis angepaßt. So schreibt auch Blümml zu ihrer Legitimation in seinem Vorwort:

Die Anordnung der "Denkwürdigkeiten" ist eine chronologische. Schrittweise können wir Pichlers Leben gleichzeitig mit den Ereignissen, die in ihrer Umwelt vorgehen, verfolgen. Alles entwickelt sich folgerichtig. Abschweifungen oder Einstreuungen, wie etwa die Abhandlung über das Gebet, treten selten auf und der chronologische Gang der Handlung erleidet hauptsächlich nur an zwei Stellen, wo sie über Grillparzer und Bauernfeld in einem Zug

in seiner 1989 veröffentlichten Monographie: "Aus [...] sozialhistorischen Interesse resultiert seit Beginn der siebziger Jahre auch die Beschäftigung mit Autobiographien sozialer Rand- und Sondergruppen, deren Aufstieg und Identitätssuche an ausgewählten Gattungsbeispielen demonstriert werden: so wird in Amerika die Black Autobiography entdeckt, in Deutschland erwacht ein neues Interesse an den Arbeiterlebenserinnerungen, und analog dazu ist die spezifische Aufmerksamkeit auf die Autobiographien von Frauen motiviert, deren gesellschaftliche Emanzipation man an der Geschichte ihrer Lebensdarstellung unmittelbar abzulesen hofft" in: Günter Niggl (Hg.): Die Autobiographie. Zu Form und Geschichte einer literarischen Gattung, Darmstadt 1989, S. 9.

9

Katherine Goodman: Dis/Closures: Women's Autobiography in Germany Between 1790 and 1914, New York 1986.

10

Elke Ramm: "Warum existieren keine 'klassischen' Autobiographien von Frauen?" In: Geschriebens Leben: Autobiographik von Frauen, Berlin 1995, S. 130-141.

Über Frauenautobiographien in dem Zeitraum 1771-1810 informieren: Helga Gallas und Anita Runge: Romane und Erzählungen deutscher Schriftstellerinnen um 1800. Eine Bibliographie, Stuttgart 1993; Eda Sagarra: "Quellenbibliographie autobiographischer Schriften von Frauen im deutschen Kulturraum 1730-1918" in: IASL 11 (1986), S. 175-231. 
berichtet, Unterbrechungen. Karoline Pichler hat eben auch hier im Formellen die Dichterin nicht verleugnen können und die Meisterschaft der Darstellung ähnlich wie Goethe bekundet . ${ }^{11}$

Blümml zielt nicht nur einen Vergleich mit Goethes Dichtung und Wahrheit an, ihm geht es vor allem um Pichlers Stil, der sich, im Gegensatz zu ihrer Kollegin Lulu Thürheim (1788-1864), an männlichen Vorbildern orientiert und somit einen künstlerischen Anspruch zu erheben berechtigt ist:

Karoline Pichler [befindet sich] durch ihre Art der Darstellung in Vorteil, denn sie bietet ein abgerundetes Ganze, das künstlerisch als solches wirkt, während die Thürheim, die Verarbeitetes mit Tagebuchblättern bunt durcheinandermengt, durch diese Darstellungsweise etwas Zerrissenes und Zerfahrenes an sich hat, das den künstlerischen Genuß. stört, wenn auch die Unmittelbarkeit und das Persönliche dadurch oft besser zum Ausdruck gelangen. ${ }^{12}$

Der Vergleich mit Thürheims Autobiographie belegt nur zu deutlich, daß Frauen eigene autobiographische Ausdrucksformen gewählt haben, und sich ihre Werke daher nicht mit den von Männern etablierten Gattungskriterien beurteilen lassen. Thürheim widersetzt sich der Norm, indem sie nicht für die Öffentlichkeit bestimmte und unbearbeitete Dokumente in ihre Autobiographie einbaut und erfüllt damit das Gattungsgebot der Selbstoffenbarung. Pichler hingegen hält am vorherrschenden Modell fest und vernichtet im Gegensatz zu Thürheim ihre Tagebuchaufzeichnungen und damit jede Spur ihres Inneren. Dennoch bezeichnet sie ihr Werk als ein "Selbstbekenntnis", daß zeigen soll "wie ich das geworden, was ich war, durch welche Einwirkungen, Umgebungen, Belehrungen, Irrtümer und Hindernisse mein Geist und Gemüt die Richtung erhalten haben, die ihnen jetzt eigen ist." ${ }^{13}$ Obwohl ihre Entwicklung das Anliegen der Darstellung sein soll, zeigt doch die Wortwahl wie sehr diese von gesellschaftlichen Ansprüchen geprägt ist. Pichler versteht

11 12 13

Blümml, "Einleitung", S. LXXVI.

2 Blümml, "Einleitung", S. LXXVI.

${ }^{13}$ Denkwïrdigkeiten I, S. 4. 
sich als ein Produkt ihrer Zeit wenn sie ergänzend schreibt: "Bei diesen Auseinandersetzungen müssen Personen, Bücher, Zeitumstände und vor allem Zeitgeister geschildert werden" ${ }^{14}$ und bezieht sich damit auf ihr männliches Vorbild Goethe, bei dem es heißt:

Denn dieses scheint die Hauptaufgabe der Biographie zu sein, den Menschen in seinen Zeitverhältnissen darzustellen, und zu zeigen, inwiefern ihm das Ganze widerstrebt, inwiefern es ihn begünstigt, er sich eine Welt- und Menschenansicht daraus gebildet und wie er sie, wenn er Künstler, Dichter, Schriftsteller ist, wieder nach außen abspiegelt. ${ }^{15}$

Was Pichler und Goethe verbindet, nämlich ihr historisches Selbstverständnis, unterscheidet sie aufgrund ihrer geschlechtsspezifischen Rolle in der Gesellschaft: bei Goethe predominiert die Wechselwirkung von Ümwelt und Ich, Pichlers eingeschränktes Wirkungsbedürfnis läßt hingegen ihre passive Grundhaltung erkennen. Während es Goethe möglich war, Erlebtes mit Wirken zu verbinden, leitet Pichler ihre Entwicklung von der sie bestimmenden Gesellschaftstruktur ab. Da ihr kein Wirkungsund damit auch kein Interpretationsfreiraum zusteht, bezieht sie sich detailliert auf familiäre und historische Geschehnisse. Das führt in ihrer Autobiographie zu einer kategorischen Wahrheitstreue, die sie veranlaßte, Goethes freizügigen Umgang mit der (Er-) Dichtung zu bemängeln:

Ich halte es für Pflicht, bei einer Selbstbiographie ganz aufrichtig zu sein, insoweit es die Klugheit, welche zwar nie eine Lüge, aber Stillschweigen gebieten kann [...]. Daher dünkt mich der Titel von Goethes Werke: Wahrheit und Dichtung aus meinem Leben, eine Art Beleidigung für den Leser, der sich nun weder eine psychologische Beobachtung noch eigentliche Belehrung versprechen kann, weil er bei keiner Beschreibung, keiner Begebenheit oder Gefühlsäußerung weiß, ob sie sich wirklich so in Goethes Geist

14

Ibid.

${ }^{15}$ Johann Wolfgang von Goethe: Dichtung und Wahrheit. In: Werke. Autobiographische Schriften, (Hamburger Ausgabe), München 1982, Bd. 9, S. 9. 
oder Leben zugetragen hat oder bloß von ihm zur anziehenderen Unterhaltung seiner Leser erfunden worden ist. ${ }^{16}$

Pichler kritisiert nicht, wie ihr vorgeworfen wurde, ${ }^{17}$ das künstlerische Verdichten, sondern das Hinzudichten von Personen und Handlungen. ${ }^{18}$ Damit berührt sie einen zentralen Aspekt der Autobiographie mit dem sich Autobiographen, wie Günter de Bruyns 1995 erschienene Reflexionen "Über Wahrheit und Dichtung in der Autobiographie" belegen, noch immer beschäftigen. Jürgen Lehmann beanstandet im Vergleich zu Goethe vielleicht zu Recht Pichlers "Wahrheitsanspruch" und ihre "biedermeierliche Pflege des Details", es fragt sich jedoch, ob sich diese Detailpflege vom Biedermeier herleiten läßt, oder ob sie nicht vielmehr eine Kompensation für das Unaussprechbare ist. Pichlers Vorwurf ließe sich damit auf ihr eigenes Werk übertragen, denn wenn man bei Goethe vielleicht nicht mit Gewißheit sagen kann, ob sich Erzähltes "wirklich" so zugetragen hat, stellt sich bei Pichler doch immer die Frage, was sie dem Leser aus politischen oder gesellschaftlichen Gründen verschweigt.

Pichlers einleitende und im ersten Druck zensierten Worte zeigen, daß sie sich nur ungern den Zensurbestimmungen anpaßte:

Sollten bis zu meinem Tode die Umstände sich ändern und wieder einige Gedanken und Preßfreiheit bis dahin in Österreich möglich sein, so steht es der Willkür meiner hinterlassenen Lieben frei, welchen Gebrauch sie von dieser Arbeit machen wollen, die ihnen gewidmet ist.

16

Denkwürdigkeiten I, S. $169 \mathrm{f}$.

17

Jürgen Lehmann greift Pichlers Urteil über Goethe an: "Solche Aussagen sind [...] Bestandteil einer bewußten Absage an Ambitionen, mit Hilfe literarischer Darstellungstechnik Selbsterlebtes zu einem Kunstgebilde zu gestalten." In: Jürgen Lehmann: Bekennen, erzählen, berichten: Studien zu Theorie und Geschichte der Autobiographie, Tübingen 1988, S. 164. 18

Aus dem gleichen Grund lehnte sie auch Bettine von Arnims autobiographischen Roman Goethes Briefwechsel mit einem Kinde ab, den sie dennoch "unendlich geistreich, voll blendender Auffassungen, voll ergreifender Stellen, voll scharfsinninger Bemerkungen" findet. "Über Wahrheit gegen die Welt und gegen sich selbst". In: Lena Jansen: Karoline Pichlers Schaffen und Weltanschauung im Rahmen ihrer Zeit, Graz 1936, S.136. 
Bereits die Aufführung ihres Dramas Ferdinand II. wurde trotz ihres persönlichen Einsatzes und einer Vorsprache bei Metternich untersagt. Die Auseinandersetzung mit der Zensurbehörde hat sie dazu veranlaßt, sich in späteren Werken an die Verordnungen zu halten. Ihre eigene Überarbeitung der Denkwürdigkeiten war nach den Bestimmungen der Zensur noch nicht ausreichend. Als Charlotte von Pelzeln sich nach dem Tod ihrer Mutter zur Veröffentlichung entschloß, wurde das Werk erst von dem Herausgeber Ferdinand Wolf durchgesehen. Darauf gelang es zum k.k. Zentral-Bücher-Revisionsamt, das folgenden Zensurzettel ausstellte: "Die Memoiren der Fr. v. Pichler sind als Mittheilungen und Ansichten einer achtbaren Frau und Schriftstellerin zu berücksichtigen, demungeachtet erscheinen nachfolgende Veränderungen und Weglassungen durchaus notwendig." ${ }^{19}$ Eine weitere Durchsicht war jedoch nötig, bevor die Druckerlaubnis gegeben wurde. Gestrichen wurden in dem von Metternich unterzeichneten Schreiben diesmal "ihre Ansichten über manche politischen Fragen, welche während ihres Lebens entschieden wurden". Unterbleiben sollten weiter Pichlers Ausführungen über das Aufführungsverbot ihres Dramas Ferdinand II.: "Freilich wäre es noch besser, wenn die umständliche Erzählung des Faktums, von dem hier die Rede ist, ganz unterbliebe und der Umstand, daß die Aufführung des Stückes Ferdinand II. auf der Hofbühne nicht gestattet wurde, nur in Kürze und mit Weglassung aller verletzenden Details bezeichnet werden wollte." 20

Das Niedergeschriebene ist - auch ohne Zensur - immer nur eine Auswahl, ein Ausschnitt aus dem erlebten Ereignis, aus dem Leben des Autobiographen. Der Autor schafft daher auch bei noch so großem Bemühen, Geschehnisse der Wahrheit getreu wiederzugeben, eine zweite Realität, die in der Autobiographie-Forschung gerne mit Schaffung eines zweiten Ichs erklärt und bezeichnet wird. Frauen habe dieses Ich den gesellschaftlichen Erwartungen, die an sie gestellt wurden, angepaßt. In der feministischen Literaturwissenschaft wurde dieses Phänomen der "Verdopplung" ${ }^{21}$ unterschiedlich benannt. Die Metapher des Spiegels be19 20

${ }^{21}$ Vgl. Elisabeth Lenk: "Die sich selbst verdoppelnde Frau." In: Ästhetik und Kommunikation 7 (1976), H. 25, S. 87. 
schreibt das "weibliche Selbstverständnis unter der Kontrolle des männlichen Blicks", der schielende Blick den Versuch, das eigene Selbstbild mit den vorherrschenden männlichen Vorstellungen zu vereinen, das Bild der Maske, das bewußte Verbergen des Ichs hinter tradierten Weiblichkeitsbildern. ${ }^{22}$ Während nun Pichlers auf die politische Zensur zurückzuführenden Auslassungen relativ einfach nachzuvollziehen sind, ist das hinter der Maske sich verbergende Ich weitaus schwerer aufzudecken.

Karoline Pichler läßt ihre Denkwürdigkeiten mit der Lebensgeschichte ihrer Mutter beginnen. Die Mutter, Charlotte von Greiner, wurde als junge Waise von Maria Theresia aufgenommen, erhielt eine umfassende Ausbildung und trat früh als Vorleserin in den Dienst der Kaiserin. Pichler war sehr stolz auf die Verbindung der Familie mit dem Kaiserhaus und berichtet ausführlich über Ereignisse, die das enge Verhältnis zwischen ihrer Mutter und der Herrscherin bezeugen. Mit Bewunderung bezeichnet sie ihre Mutter als "eine der geistreichsten und der selbständigsten Frauen [...], die nur je vorgekommen", ${ }^{23}$ die sich, ihrem ernsten und männlichen Geist ${ }^{24}$ gemäß für "Naturgeschichte, Naturlehre, sogar Astronomie" interessierte und danach strebte "durch die Bekanntschaft mit den Religionen und Mythen aller alten und neuen Völker, mit den Traditionen, den Geschichten der Vorwelt, den Mysterien, Tempelgebräuchen usw. zur ursprünglichen und höchsten Erkenntnis in Rücksicht der Gottheit, unseres Verhältnisses zur ihr, der Geologie und Kosmologie zu gelangen". ${ }^{25}$ Der Vater hingegen hatte einen Hang zur Kunst, zur Dichtung und vor allem zur Musik und einige Jahre nach ihrer Verheiratung wurde ihre Wohnung Treffplatz für "die meisten berühmten Männer jener Periode", Pichler nennt Sonnenfels, Denis, Abbate Metastasio, Mastalier, Haschka und Alxinger. Schon als Kind durfte sie an diesen Abendzirkeln teilnehmen, saß mit Mozart am Klavier und schreibt: "Heiteres Gespräch, literarische und politische Neuigkeiten,

22

Sigrid Weigel: "Der schielende Blick" in: S. Weigel und I. Stephan (Hgg.): Die verborgene Frau. Sechs Beiträge zu einer feministischen Literaturwissenschaft, Berlin ${ }^{3} 1988$, S. 83-137.

23

Denkwürdigkeiten I, S. 299.

24

25

Denkwürdigkeiten I, S. 172.

${ }^{5}$ Denkwürdigkeiten I, S. $46 \mathrm{f}$. 
alles, was im Gebiete der Künste [...] Neues erschien, ward bei uns gezeigt, gelesen, oder doch besprochen. ${ }^{26}$

Die Eltern legten auf eine umfassende Erziehung ihrer Tochter großen Wert. Sie erkannten ihr dichterisches Talent, das sie auch, wie es die Veröffentlichung eines Gedichtes der Zwölfjährigen im Wiener Musenalmanach $^{27}$ zeigt, unterstützten. Sie hielten sie aber auch zum Studium der Mathematik an, um ihre "wilde Phantasie" zu zügeln. Pichler versteht rückblickend, daß die Eltern richtig gehandelt haben, da sie so gelernt habe, "richtig" zu denken.

Das Elternhaus hat ihr jedoch mehr als eine gute Allgemeinbildung vermittelt. Die Mutter hat sie, wie folgendes Zitat belegt, in die feministischen Ideen der Zeit eingeweiht:

Das männliche Geschlecht kam bei [den] Untersuchungen [meiner Mutter] nicht zum besten weg, und meine Mutter war sehr geneigt (wie ich später hörte, als ich imstande war, solche Begriffe zu fassen, und ihr oft Bücher vorlas, welche in diesem Sinne geschrieben waren, z.B. Sur les droits des femmes, par Mme de Wolstonecraft) das System aufzustellen, daß die Frauen ursprünglich von der Natur und Vorsicht zur Herrschaft bestimmt seien, und dieses Vorrecht durch eine Art Usurpation des männlichen Geschlechts, welches uns an physischen Kräften übertrifft, verloren habe. Doch das ist eine Abschweifung. ${ }^{29}$

Es ist bezeichnend, daß Pichler zwar die radikalen Ansichten der Mutter erwähnt, sie jedoch mit dem abschließenden Satz als nebensächlich abtut. Während sie einerseits den "männlichen Geist" ihrer Mutter bewundert, nimmt sie anderseits von ihren Ideen Abstand und bemüht sich, indem sie in der Folge das häusliche Pflichtbewußtsein der Mutter betont, ihre Weiblichkeit in den Vordergrund zu stellen. Pichlers Tendenz, Gescheh-

26

Denkwürdigkeiten II, S. 399.

27

Wiener Musenalmanach auf das Jahre 1782, S. 163 ff. "Auf die Genesung meiner Freundin" in: Blümml, Denkwürdigkeiten I, S. 456. 28 29

Denkwürdigkeiten I, S. 38.

Denkwürdigkeiten I, S. 46f. 
nisse, die das Weiblichkeitsbild der Frau negativ beeinflussen könnten, zu korrigieren, zeigt sich auch in ihren Beschreibungen kleinerer Vorfälle. So vertuscht sie z.B. das Verhältnis zwischen ihrer Mutter und dem zehn Jahre jüngeren Haschka, der ihr Hauslehrer war: "Herr Haschka [hatte] infolge mancher kleinen Mißverständnisse unser Haus verlassen, obgleich er uns immerfort und fleißig besuchte." ${ }^{30}$

Pichlers Bemühen, die feministischen Ansichten der Mutter zu decken, gibt über ihre eigenen Darstellungen Aufschluß. Sie schreibt über ihre erste größere Veröffentlichung, die Gleichnisse:

Um diese Zeit (1800) ungefähr fand mein Mann, als er eines Tages in meinen Schriften herumsuchte, das Manuskript meiner Gleichnisse [...]. Sie gefielen Pichlern, und zwar so sehr, daß er mir den Vorschlag tat, sie der Welt durch den Druck zu übergeben. Vor diesem Gedanken erschrak ich im eigentlichsten Sinne; denn wenn gleich einzelne kleine Gedichte von mir gelegentlich allein oder in Almanachen erschienen waren, so hatte ich nie daran gedacht, als Schriftstellerin mit einem eigenen Werke aufzutreten [...]. [Mein Mann] schlug mir vor, das Manuskript, ehe wir den großen Schritt vor die öffentliche Meinung täten, einigen vertrauten [...] Freunden (das waren u.a. Haschka, Sonnenfels) zu zeigen. Das einstimmige Urteil dieser Herrn fiel günstig und ermunternd aus, und so erschienen denn, obgleich von mir noch immer mit Angst und Sorge aus dem schützenden Schatten der Unbekanntheit entlassen, die Gleichnisse, und ich trat öffentlich vor der Welt als Schriftstellerin auf. ${ }^{31}$

Die Gleichnisse, die Pichler ursprünglich nicht für die Öffentlichkeit bestimmt einer Freundin gewidmet hatte, werden mit dieser Erklärung sowohl von ihrem Mann als auch von anerkannten Schriftstellern legitimiert. Mit dieser Veröffentlichung begann nicht nur Pichlers öffentliches Leben als Schriftstellerin, es war auch der Anfang ihrer eigenen Gesellschaftsabende, der Greinersche Salon hatte sich nach dem Tod des Vater und dem Umzug der Familie in die Vorstadt aufgelöst.

30

Denkwürdigkeiten I, S. 75.

31

Denkwürdigkeiten I, S. $226 \mathrm{f}$. 
Die (scheinbare) Unterstellung unter die männliche Autorität läßt sich an vielen Stellen beobachten. In einem Brief bittet sie einen Geschichtsforscher, recht streng in der Vorrede zu ihrem Schauspiel "Ferdinand II.", jede "geschichtliche Unrichtigkeit, die sich wohl leicht in eine Frauenarbeit eingeschlichen haben mag" zu rügen und ihr gefällig zu sagen, ob und wie sie diese verbessern könne ${ }^{32}$ und schützt sich so vor der öffentlichen Kritik. Schon bei ihrem ersten Drama, dem männlichen Genre par excellence, benutzte sie diese Verteidigungstechnik und gibt an, daß ihr Mann sie zu der Verfassung einer Bühnendichtung überreden mußte. Mehrfach betont sie dann auch noch, daß Frauen sich nicht "auf den Kothurn wagen" sollten, was sie jedoch nicht davon abhält, weitere Tragödien zu schreiben. Auffallend ist nun, daß Andreas Pichler, der das Werk seiner Frau so gefördert haben soll, in den Denkwürdigkeiten nirgends literarisches Interesse zugesprochen wird, und er auch am Leben des Salons nicht teilgenommen hat.

Wie sehr sie jedoch selbst in den praktischen Teil der Veröffentlichungen ihrer Werke involviert war, zeigen Zeugnisse, die nicht für die Öffentlichkeit bestimmt waren. So erwähnt Pichler beispielsweise nie ihr Honorar, das an ihrem Erfolg gemessen, nicht unerheblich gewesen sein kann. Auch belegt ihr Testament, daß sie sehr genaue Vorstellungen von $\mathrm{zu}$ fordernden Honoraren hatte. Auseinandersetzungen mit Verlegern zeigen ebenfalls, daß sie an Verhandlungsprozessen aktiv beteiligt war. Interessant ist in diesem Zusammenhang ein Brief an Therese Huber, der Pichlers Ärger über einen Kollegen bezeugt. Gemeinsam mit ihm hatte sie an einem Aufsatz gearbeitet, für den sie lange (unweibliche) Nachforschungen angestellt hatte und will nun, nachdem er sich kurz vor der Veröffentlichung zurückgezogen hat, den herrenlosen Artikel nicht fahren lassen. Sie entschließt sich daher, die Redaktion in die Hand zu nehmen und den Artikel anonym zu veröffentlichen.

Anonyme Veröffentlichungen sind ungewöhnlich bei Pichler. ${ }^{33}$ Die 32 Jansen, S. 169.

33

Elke Ramm zitiert in ihrem Artikel die Untersuchung von Gallas/Runge, nach der um 1800 dreihundertsechsundneunzig Veröffentlichungen von Romanen und Erzählungen von Frauen zweihundertzweiundfünfzig anonym erschienen, sieben kryptonym und sieben pseudonym. Ihren eigenen Recherchen zur Frauenautobiographie aus dem achtzehnten und beginnenden neunzehnten Jahrhundert liegt ein Textkorpus von ca. zeihundert auto- 
Wienerin steht zu ihrem Namen, sie gibt sich mit ihrem vollem Namen, Karoline Pichler, geb. von Greiner zu erkennen, und ermuntert auch ihre Kolleginnen ein Gleiches zu tun. Susanne Kord sieht in dem Gebrauch des Doppelnamens eine für Frauen unübliche Überidentifizierung. ${ }^{34}$ Es ist anzunehmen, daß sich Pichler als Künstlerin mehr mit dem Elternhaus als mit ihrem Mann identifizierte und dies durch ihren Namen zum Ausdruck bringen wollte.

Nur noch bei einer zweiten Gelegenheit verschweigt sie in einer Rezension ihren Namen. ${ }^{35}$ Sie geht so weit, diese Rezension über Grillparzers Sappho in den Denkwürdigkeiten zu verleugnen und verstößt damit gegen ihr Wahrheitsgebot, um den gesellschaftlichen Anforderungen, die an sie als Frau gestellt wurden, gerecht bleiben zu können.

Das Aufdecken von Verschwiegenem ist aufschlußreich. Man sieht, daß Pichler ihr eigenes Image ganz bewußt geformt hat, indem sie alles, was ihr aufgrund ihrer schriftstellerischen Tätigkeit männliche Attribute hätte verleihen können von sich fernhält, oder aber sie legitimiert ihr Schreiben durch eine männliche Autorität. Pichlers Bestreben ihrer Betätigung weibliche Qualitäten zugrunde zu legen, kommt auch in ihrer Forderung, der männlichen Schriftstellerei ein weibliches Pendant zu geben zum Ausdruck: Erst "Achtung für echte Weiblichkeit, Häuslichkeit und Ordnung" geben "weiblicher Schriftstellerei ihren wahren Wert". ${ }^{36}$ Weiblichkeit und Schreiben sind damit kein unversöhnlicher Gegensatz mehr, sondern verschmelzen zu einer harmonischen Einheit. Schließlich legitimiert sie das Schreiben durch seine erzieherische Funktion und legt in

biographischen Schriften zugrunde (Briefe, Tagebücher, Fragmente, Memoiren, Lebensbeschreibungen, Erinnerungen). Nur elf Frauen haben ihre Autobiographie zu Lebzeiten und unter ihrem Namen veröffentlicht. 34

Susanne Kord: “'Und drinnen waltet die züchtige Hausfrau'? Caroline Pichler's Fictional Auto/Biographies" in: Women in German Yearbook 8 (1992), S. 145.

${ }^{35}$ An Therese Huber, Redakteurin des Morgenblatts am 4. Mai 1818

"Aber e i n Blatt erlauben Sie mir beizuschlißen und gönnen ihm - wenn Sie es geeignet finden, ein Plätzchen im Morgenblatt - aber ohne meinen Namen, weil ich es mir zum Gesetz gemacht habe, nie mein Urtheil über ein Kunstwerk öffentlich auszusprechen." zit. in: Brigitte Leuschner (Hg.): Schriftstellerinnen und Schwesterseelen. Der Briefwechsel zwischen There36 se Huber und Karoline Pichler, Marburg 1995.

${ }^{6}$ Denkwürdigkeiten I, S. 409. 
Aufsätzen und Romanen die gesellschaftlichen Vorteile weiblicher Bildung dar:

So bald alles, was wir lernen, üben, denken, dem höchsten Zwecke - nicht nur des Weibes sondern des Menschen untergeordnet wird dem Zwecke moralischer Veredlung, so bald das gebildetere Weib auch eben darum das bessere Weib, die verständigere Hauswirtin, die erfahrnere Erzieherin, die teuere verläßlichere Freundin des Mannes sein wird - so werden alle Klagen über die falsche Richtung und die schädlichen Folgen der höheren Cultur des weiblichen Geschlechtes wegfallen. ${ }^{37}$

Pichlers Bildungsbedürfnis erklärt sich durch ihre Erziehung. Was sie hier fordert, ist jedoch nicht mehr als eine Kompromißlösung: Der Mann und nicht die Frau profitiert von der geforderten weiblichen Bildung. Unter diesem Vorwand kann sie aber ihr Bildungsbedürfnis mit den Lebensbedürfnissen des Mannes in Einklang bringen. Dieser Verdeckungsmechanismus wird deutlich, wenn man Aussagen betrachtet, die unter anderen gesellschaftlichen Bedingungen geschrieben sind. So konnte Pichler in der Zeit der Befreiungskriege, als die allgemeine Notlage im Land eine Erwerbstätigkeit der Frau denkbar machte, ohne Kritik fürchten zu müssen, die beruflichen Vorteile für gebildete Frauen darlegen und ihre Selbständigkeit fordern: ${ }^{38}$ "Das vielseitig gebildete Mädchen wird, sie mag heiraten oder nicht, -ein vollendetes Wesen, ein ganzer Mensch sein". 39

In ihren Romanen läßt Pichler es jedoch nicht zu solchen emanzipatorischen Forderungen kommen. Hier werden Weiblichkeit und künstlerische Betätigung - bei den beispielhaften weiblichen Figuren - in Einklang gebracht. Über Leonore in dem 1818 erschienen Roman Frauenwürde heißt es:

37"Über die Corinne der Frau von Staël" (1807) zit. bei: Barbara BeckerCantarino: "Caroline Pichler und die 'Frauendichtung"' in: Modern Austrian Literature 12 (1979), No. 3/4, S. 9 .

${ }^{38} \mathrm{Vgl}$. Becker-Cantarino, S. 9.

39 "Über die Bildung des weiblichen Geschlechtes" (1810) zit. bei: BeckerCantarino. 
Sie malt wirklich mit Meisterschaft. Ich habe Arbeiten gesehen, die ihr einen bedeutenden Rang unter ihren Kunstgenossen sichern würden, wenn sie sich entschließen könnte, sich öffentlich zu zeigen. Aber davor schaudert ihre zarte Weiblichkeit zurück, so wie ihr stiller Sinn allein an den Werth ihres Talents nicht glaubt, den doch Jeder anerkennen muß, welcher ihre Arbeiten sieht. Sie scheint nur sich und ihren Lieben zur Lust zur malen. Sich in ihren Kunstgebilden zu ergötzen, zu verlieren, und ihrem Gemahl oder einigen Freuden Freude damit zu machen, ist der Endzweck ihres Fleißes. Übrigens fließt dieses bedeutende Talent nirgends störend auf ihr häusliches Leben ein. Sie weiß die Künstlerin sehr bestimmt von der Hausfrau zu scheiden, und jeder zu geben, was ihr gehört. Dennoch verschmilzt auf der anderen Seite das Eine so schön in's Andere. Sie würde minder liebenswürdig seyn als Frau, wenn ihr dieser hohe Sinn für Kunst fehlte, und minder achtungswerth als Künstlerin, wenn sie ihrem Talent auch nur Eine ihrer Pflichten opferte.

Diese Beschreibung der weiblichen Protagonistin ist in mehrfacher Hinsicht aufschlußreich. Leonores Scheu öffentlich aufzutreten, reflektiert nur allzu genau Pichlers zitierte Version der Veröffentlichung ihrer Gleichnisse. In einem Brief an ihre Freundin Therese Huber wird jedoch deutlich, daß sie es im Gegenteil unterstützt, daß Frauen in die Öffentlichkeit treten und unter ihrem Namen publizieren. ${ }^{41}$ Weiterhin sind Parallelen zu der eingangs von Reinbeck zitierten Stelle zu erkennen. Auch er zeigt unter Betonung von Pichlers "edlen Weiblichkeit", daß weibliche Berufstätigkeit und Hauswesen vereinbar sind. Während aber Reinbeck die beiden Aufgabenbereiche der Frau trennt, setzt Pichler die Weiblichkeit in Wechselwirkung mit Leonores künstlerischer Begabung. Pichler zeichnet in ihren Romanen nicht nur vorbildhafte Charaktere, sondern auch - in ebenso erzieherischer Absicht - abschreckende Beispiele. Es ist nun interessant zu sehen, daß in diesem Roman eine Frau, Rosalie von Sarewsky, diese Rolle übernimmt, die auffallende Ähnlichkeit mit ${ }^{40}$ Frauenwürde. In: Pichlers Schriften, Stuttgart 1828, Bd. 28, S. 41 .

${ }^{41}$ So am 11. Jänner 1827. "Mich freut es daß Sie sich jetzt zuweilen unterzeichnen." zit. in Leuschner, S. 131. 
Pichler selbst hat. So erfährt man über ihre Erziehung:

Shakespeare und Dante, Milton und Klopstock, Rousseau und Calderon wurden in ihrer Mundart gelesen, die Tragiker der Römer und Griechen machten treffliche Übersetzungen mir verständlich, und so schwelgte mein Geist in hohen Genüssen, in einem Alter, wo sonst noch der dumpfe Kindersinn einigen helleren Anschauungen zu weichen beginnt.

Rosalie von Sarewsky ist nicht nur Dichterin und hat eine ähnliche Erziehung genossen, sie lebt, wie sich herausstellen wird, das aus, was Pichler aufgrund ihres Tugendideals verschweigen mußte.

Nach Lena Jensen hat Germaine de Staël dieser Figur Modell gestanden. Pichler bewunderte die Französin, die in ihrem Land als Schriftstellerin gesellschaftliche Anerkennung gefunden hatte. So wurde ihr, als die berühmte "Madame" in Wien war und sie in ihrem Salon besuchte, der Kreis der tugendhaften Frauen auf einmal sehr unangenehm.

[Madame de Staël] saß neben meiner Mutter auf dem Kanapee, ich nahm meinen Platz an ihrer Seite, Schlegel, Hammer und Collin näherten sich ebenfalls, die Frauen rings um den Tisch hatten ehrerbietig gegrüßt und sich jetzt wieder niedergesetzt, um - zu stricken [...]. Mich überfiel eine Art von Bangigkeit, so oft ich auf diese schweigsame Gesellschaft sah, die die hochberühmte Frau lautlos umgab, sie nur dann und wann mit neugierigen Blicken musternd, und mir dachte, welche Vorstellung sich Frau von Staël wohl nach diesem Abend von dem Kreis machen möchte, in dem ich lebte.

Mit dieser Bemerkung wird deutlich, daß Bildung ein wesentlicher Bestandteil ihres Weiblichkeitsideals ist. Interessant ist nun, daß Pichler diese Aussage zu einem Zeitpunkt macht, in dem eben diese weibliche Bildung durch die Anwesenheit der "hochberühmten Frau" legitimiert ist und sich damit das oben angesprochene Verhaltensmuster wiederholt.

Frauenwürde, Bd. 29, S. 73.

43

Denkwürdigkeiten I, S. $319 \mathrm{f}$. 
In einem weiteren Kreis, auf den bereits Susanne Kord aufmerksam gemacht hat, unter ihren Freundinnen, kann sich Pichler ohne ihre Weiblichkeitsmaske zeigen. Freundschaften und vor allem Freundschaften mit Frauen waren für Pichler sehr wichtig und spielten eine besondere Rolle in ihrem Leben. Schon ihr erstes Gedicht war an eine Jugendfreundin gerichtet, die Gleichnisse einer weiteren Freundin gewidmet. Freundschaft galt ihr "als ein Bund für das Leben und noch weiter hinaus, dessen Zweck gegenseitige Vervollkommnung war". Die Freundin sollte sie "ganz erkennen, beurteilen, ermahnen, tadeln, mit einem Wort, bessern können, sowie [sie] das gleiche bei ihr zu tun bereit war" ${ }^{44}$ und ihr konnte anvertraut werden, was der Öffentlichkeit, dem Leser der Denkwürdigkeiten verschwiegen werden mußte. Pichler führte viele Brieffreundschaften, unter anderem mit Therese Huber, ${ }^{45}$ die sie jedoch nie persönlich kennengelernt hatte.

Besondere Bedeutung in ihrem Leben haben daher die ebenfalls bei Kord erwähnten alljährliche Urlaube Pichlers auf dem ungarischen Gut ihrer Freundin Maria von Zay in den Jahren 1814-1829. Das Reisen wird hier nicht mehr, wie zu anderer Stelle beklagt. Auch werden diese Aufenthalte in den Denkwürdigkeiten nur kurz erwähnt, man erfährt nur, daß ihr Mann sie jeweils abholte. Hier also trafen sich die Freundinnen - Therese von Artner, Maria von Zay, Marianne von Neumann-Meissenthal, Luise Brachmann -, sie waren allein unter sich und aus Briefen geht hervor, daß Pichler hier die glücklichste Zeit ihres Lebens verbrachte. Die Freundinnen, die sich im Briefwechsel "Schwestern in Apollo" nannten, arbeiteten gemeinsam und lasen sich ihre Stücke vor. ${ }^{46}$ Ihrer Freundin Therese von 44

Denkwürdigkeiten I, S. 138. 45

In einem Brief an Therese Huber heißt es z.B.: "Ich weiß nicht, ob ich Recht habe, aber mich dünkt, wir Weiber sind in unserer Entwicklung weiter geschritten als verhältnismäßig die Männer." zit. in: Leuschner, S. 130.

Es überrascht nicht, daß Pichler auch ihre Freundinnen mit dem Bild der edelsten Weiblichkeit beschrieb. Während Luise Brachmann z.B. von zeitgenössischen Kritikern als "schwärmerisch", "leidenschaftlich", "unglücklich" und auch "wahnsinnig" bezeichnet wird, schreibt Pichler, daß die Freundin zu "keiner heftigen Leidenschaft fähig" gewesen sei, zu ihrem Selbstmord sagt sie, "daß keine augenblickliche leidenschaftliche Spannung, [...] sie zur Verzweiflung trieb, sondern langsam, aber lange wirkende, drückende, enthmuthigende Verhältnisse und vielleicht körperliches Übelfinden die nächste Ursache ihres Todes waren." Therese von Artners 
Artner widmete sie folgendes Gedicht:

An meine Freundin Theone

Und mir öffnet so weit die Brust, und süße Gefühle

Ziehen durch Blick und Ton hell in die Seele mir ein.

Wunderbar reget es sich in des Innersten Tiefen. Gestalten

Blühen auf und vergeh'n, Stimmen erklingen und flieh'n

Helle Gedanken strömen empor aus dem wallenden Busen,

Und die Worte, sie reih'n sich wie von selber zum Lied.

Alles rings um mich her vergessend, murmelt die Lippe,

Was in dem tiefen Gemüt mächtig sich reget und glüht. ${ }^{47}$

Nach einer solchen Selbstoffenbarung wird man in den Denkwürdigkeiten und in Romanen umsonst suchen. Nur in ihrem Freundschaftskreis konnte Pichler sich von dem sich auferlegten Weiblichkeitsbild befreien und ihren wahren Gefühlen Ausdruck verleihen. In Frauenwürde läßt sie hingegen die vorbildliche Leonore solches Gedicht verurteilen:

Noch einmal las ich ihr letztes Gedicht, das sie vor Kurzem gedichtet hatte. Jetzt verstand ich erst ganz die düstere Glut desselben. Der Pinsel ist in Feuer getaucht, aber es ist eine verzehrende Flamme; und so schön es ist, so hätte ein Weib es entweder nie machen, oder nie drucken lassen sollen. Wie kann man seine Gefühle, seine Verlassenheit so der Welt [...] kund geben ${ }^{48}$

Die Gegenfigur Rosalie von Sarewsky kommentiert als Verfasserin des Gedichts aus ihrem innersten Gefühl:

\footnotetext{
"Schreibwut" und ihre unkonventionelle Weigerung zu heiraten, bemüht sich Pichler folgendermaßen zu korrigieren: "Ihr reichgeschmückter Geist, ihre mannigfachen Talente, besonders ihre ausgezeichnete Dichtergabe entfremdeten sie auf keine Weise ihren weiblichen Beschäftigungen. Mit Besonnenheit und Einsicht waltete sie im Hauswesen, an welchem sie thätigen Antheil nahm, am Nähtische, am Stickrahmen. [...] und doppelt ist es bey diesen liebevollen Anlagen zu verwundern und zu bedauern, daß Therese sich nie entschlossen, einen Mann mit ihrer Hand zu beglücken." (TvA 194202). Vgl. Kord, S. 147-149.

47

SW 23:5ff., zit. bei Kord, S. 152. 48

Frauenwïrde, Bd. 29, S. 66.
} 
Jenes Gedicht ist mir gelungen. Es hat seinen Zweck erreicht. Was die Welt davon denkt, gilt mir gleich. Die Unparteiischen bewundern es, und die Bosheit findet überall Gelegenheit, Gift zu saugen. Sie findet es zu individuell, und will deuten, auslegen. Mag sie doch! Keines dieser absprechenden Gemüther vermag in der heißen Flamme zu glühen, die diese Töne aus dem Innersten meiner Brust, gleich Lavaströmen aus den Tiefen des Vulkans, hervortreibt, und die dunkeln Geheimnisse der leidenden Seele in halbverstandenen Lauten kund gibt. ${ }^{49}$

Pichler hat mit dieser Figur ein Gegenbild zu ihrem postulierten Weiblichkeitsideal geschaffen und es ist nicht auszuschließen, daß sie in ihr ihre unterdrückten Gefühle zum Ausdruck bringt. Einer Freundin dieses Kreises, Maria von Zay, gesteht sie in einem Brief, daß "Phantasie und reizbares Gefühl" ein himmlisches - aber ein gefährliches Geschenk" seien. Was sie innerlich dagegen zu kämpfen habe, wie sie sich auch damals noch "in weit vorgerückten Jahren, so leicht und so tief aufgeregt fühle", wie dann ihre ganz Seele in Aufruhr" komme, das wisse meistens nächst Gott, den sie um Beistand gegen sich selbst anflehe, nur sie allein. ${ }^{50}$ Dieses letzte Zitat belegt nur allzu deutlich den inneren Kampf, den Karoline Pichler zwischen Pflicht und Neigung auszutragen hatte. Hier sieht man, wie sehr sie sich die Harmonie, die sie in den Denkwürdigkeiten darzustellen sich bemüht hatte, erkämpfen mußte.

Mit den Befreiungskriegen war für Karoline Pichler eine Zeit gekommen, in der sie öffentlich ihre Gefühle zeigen konnte, in der sie öffentlich zu ihrem Dichtertum stehen konnte. In diese Zeit fällt auch der Höhepunkt ihres schriftstellerischen Erfolgs. Sie berichtet über diese Zeit: "Hier in Wien war alles freudig und in begeisterter Stimmung. Man dachte daran, die frohen Ergebnisse auf alle Weise zu feiern, und mir wurde der Antrag gemacht, eine Kantate: "das befreite Deutschland" zu dichten, welche Spohr in Musik setzen sollte [...] Überhaupt war mein Gefühl und meine Phantasie damals sehr angeregt, und ich dichtete viel." ${ }^{51}$ Und anläßlich 49 Frauenwürde, Bd. 29, S. 51. 50

18. März 1826. Ungedr. Brief Nr. 462 zit. bei: Jansen, S. 391. 
des Erfolges ihres Stückes "Heinrich von Hohenstaufen" heißt es: "Die Versammlung war zahlreich und glänzend, der gesamte Hof erschien in kaiserlichen Logen [...] Am Schlusse des Stückes wurde heftig applaudiert und die Dichterin gerufen." ${ }^{52}$ Nach der Premiere im Oktober 1813 wurde das Stück, das am königlichen Theater in Berlin und auch in Weimar unter Goethes Leitung aufgeführt wurde bis Anfang 1818 siebenundzwanzigmal gespielt.

Die Zeit der Befreiungskriege bedeutete für Karoline Pichler sowohl innerlichen als auch äußerlichen den Höhepunkt ihrer Karriere als Schriftstellerin. Nun konnte die Patriotin ihrer "Phantasie" freien Lauf lassen, sie wurde bejubelt, gefeiert, man forderte sie auf, weitere Stücke zu schreiben. Sie mußte sich nicht mehr für ihre Phantasie entschuldigen, sie mußte sie nicht bändigen, sie muß sie nicht mehr in ein harmonisches Einverständnis mit ihrer Weiblichkeit bringen. Sie konnte die Maske abnehmen und ein inneres Gefühl, ihren tiefempfunden Patriotismus, mit der Öffentlichkeit teilen.

Mit der Restauration wurde es jedoch wieder still um Pichler. In einem Brief an Streckfuß schreibt sie 1828 "Man unterdrückt seine Gedanken, weil man nicht weiß, wer da aufhorcht und berichtet" ${ }^{53}$ Das hatte auch Auswirkungen auf Pichlers Abendgesellschaften, zu denen sich immer weniger Leute einfanden. Eine neue Zeit brach an, es war das Ende der Schriftstellerin und der Gesellschafterin. Pichler nahm sich 1843 das Leben. Dieser Akt, den sie ihr Leben lang bei Zeitgenossen und auch bei ihrer Antiheldin Rosalie von Sarewsky heftig verurteilte, offenbart sich als letzter Widerspruch ihres Lebens- und damit ihres Weiblichkeitsentwurfes.

Karoline Pichler war eine der wenigen Frauen ihrer Zeit, die es geschafft haben, aufgrund ihrer eigenen Leistung und nicht weil sie die Frau, Tochter oder Schwester eines berühmten Mannes war, öffentliche Anerkennung von ihren Zeitgenossen zu erhalten. Sie hat es geschafft, weil sie die von ihr geforderte gesellschaftliche Rolle angenommen hat. Dieses Rollenverhalten hat sie nicht nur vor Angriffen von ihren 51

Denkwürdigkeiten II, S. $8 \mathrm{f}$.

${ }^{52}$ Denkwürdigkeiten II, S. 4 f.

53

Glossy, S. 233 f. 
männlichen Kritikern geschützt, es hat ihr auch ermöglicht, ihre Ideen über die Bildungfähigkeit von Frauen zu verbreiten. Daß Karoline Pichler und ihr Werk heute in Vergessenheit geraten ist, liegt daran, das sie sich auch in ihren Romanen an gängige Verhaltensmuster gehalten hat, die sie, genau wie in den Denkwürdigkeiten nicht durchbrechen wollte.

Karoline Pichlers Denkwürdigkeiten: Ein Selbstbekenntnis? Nein, Pichler verbirgt ihr Inneres, ihr Selbst, ihre Phantasie hinter der Maske der Weiblichkeit.

Die äußere Zensur hat die innere Zensur nicht unberührt gelassen. Pichler hat ihre Spuren sorgsam verwischt und somit für die Nachwelt das Bild von sich geprägt, das ihr ihr Schriftstellerinnendasein in der Wiener Gesellschaft ermöglicht hat.

Karoline Pichlers Denkwïrdigkeiten: Ein Selbstbekenntnis, das keines werden konnte. 